\title{
Hard form factors for meson-baryon strong couplings as derived from deep inelastic lepton scattering
}

\author{
W-Y.P. Hwang ${ }^{1,2}$, J. Speth ${ }^{2}$, and G.E. Brown ${ }^{3}$ \\ ${ }^{1}$ Department of Physics, National Taiwan University, Taipei, Taiwan 10764, ROC \\ ${ }^{2}$ Institut für Kernphysik der Kernforschungsanlage Jülich, W-5170 Jülich. Federal Republic of Germany \\ ${ }^{3}$ Department of Physics, State University of New York at Stony Brook, Stony Brook, NY 11794, USA
}

Received September 6, 1990 ; revised version January 14, 1991

As stimulated by earlier attempts for obtaining the $\pi N N$ and $\pi N \Delta$ form factors from the deep inelastic lepton scattering data, we extend the analysis by taking into account effects of additional mesons including $\rho, \omega, \sigma$, $K$, and $K^{*}$, with the coupling constants fixed by the lowenergy nucleon-nucleon and hyperon-nucleon scattering data. Contrary to an earlier claim that the $\pi N N$ and $\pi N \Delta$ form factor must be very soft (e.g., with the cutoff mass less than $500 \mathrm{MeV}$ in the monopole form), we find, for example, that with all form factors parametrized in the dipole form, a universal cutoff mass of $1150 \mathrm{MeV}$ in the $\Delta / N$ sector and $1400 \mathrm{MeV}$ in the $A / \Sigma$ sector yields predictions in excellent agreement with recently published neutrino data on the momentum fractions carried by the $s, \bar{u}$, and $\bar{d}$ quarks, as well as consistent with the sea-tovalence ratio extracted from the CDHS data and the Fermilab E615 experiment. Similar results can also be obtained by using exponential cutoffs for all couplings, or by using monopole forms for some vertices while retaining dipole forms for the rest. The success of the mesonexchange picture in generating the strangeness content in a proton suggests an alternative understanding of the origin of sea quarks in the proton.

PACS: 13.75.Gx; 13.75.Jz; 13.60.Hb

In 1972, Sullivan [1] pointed out that, in deep inelastic scattering (DIS) of a nucleon by leptons, the process shown in Fig. 1, in which the virtual photon strikes the pion emitted by the nucleon and smashes the pion into debris, will scale like the original process where the virtual photon strikes and smashes the nucleon itself. In other words, the process will contribute by a finite amount to cross sections in the Bjorken limit, $Q^{2} \rightarrow \infty$ and $v \equiv E_{\ell}-E_{\ell}^{\prime} \rightarrow \infty$ with $x \equiv Q^{2} /\left(2 m_{N} v\right)$ fixed. In 1983 , Thomas [2] observed that in the Sullivan process the virtual photon will see most of time the valence distributions in the pion as the probability of the pion carrying the momentum faction $y$ of the nucleon, $f_{\pi}(y)$ to be given by $(2 \mathrm{~b})$ below, peaks at $y \approx 0.3$, a region where only valence quarks and antiquarks are relevant. By attributing to the Sullivan process the excess of the momentum fractions carried by $\bar{u}$ and $\bar{d}$ quarks as compared to that of the $\bar{s}$ quarks, Thomas was then able to set a limit on the momentum fraction carried by those pions which surround the nucleon. The limit is such that a chiral bag radius cannot be too small, say $R=0.87 \pm 0.10 \mathrm{fm}$. In 1989 , the idea of Thomas was elaborated somewhat furher by Frankfurt, Mankiewicz, and Strikman [3], who obtained, with both the $\pi N N$ and $\pi N \Delta$ couplings taken into account, that, if the $\pi N N$ coupling is parametrized by the form factor in a monopole form (see (3) below), the cutoff mass must be less than $0.5 \mathrm{GeV}$, a reminiscent of having large bag radius.

Very recently, there is new information [4] obtained by the CCFR Collaboration at Fermilab which, as we shall see, is highly relevant for the argument of Thomas. Denoting $\langle x\rangle_{i}$ the momentum fraction carried by partons of flavor $i$, the CCFR Collaboration obtained, with $\left\langle Q^{2}\right\rangle=16.85 \mathrm{GeV}^{2}$,

$\kappa \equiv \frac{2\langle x\rangle_{s}}{\langle x\rangle_{i \bar{u}}+\langle x\rangle_{d}}=0.44_{-0.07-0.02}^{+0.09+0.07}$,

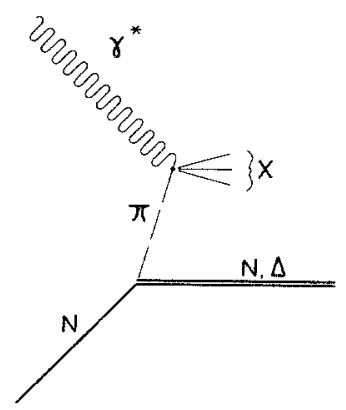

Fig. 1. The original Sullivan process which scales in the Bjorken limit 
$\eta_{s} \equiv \frac{2\langle x\rangle_{s}}{\langle x\rangle_{u}+\langle x\rangle_{d}}=0.057_{-0.008-0.002}^{+0.010+0.007}$,

$R_{\bar{Q}} \equiv \frac{\langle x\rangle_{\tilde{u}}+\langle x\rangle_{\bar{d}}+\langle x\rangle_{s}}{\langle x\rangle_{u}+\langle x\rangle_{d}+\langle x\rangle_{s}}=0.153 \pm 0.034$.

Here (1c) comes from an earlier measurement with errors determined from cross section results [5]. As one would expect $\langle x\rangle_{\bar{s}} \approx\langle x\rangle_{s}$ inside a nucleon, the value for $\kappa$ (together with the antiquark distribution of Field and Feynman for a free proton [6]) constitutes the basis for the argument of Thomas [2,3]. Here the lower value reported for the ratio may loosen slightly the bounds obtained earlier [2,3], but not by any significant amount.

Assuming the standard wisdom that gluons carry (45-50)\% of the nucleon momentum, we obtain from (1a)-(1c) that the total momentum fraction carried by antiquarks of all kinds must be in the vicinity of (6-7) \% while $\langle x\rangle_{s} \approx\langle x\rangle_{s} \approx 1 \%$. These values will play an important role in the following calculation. The basic difference between the previous approaches [2,3] and ours is that we introduce kaons as is required [7] by the hyperon-nucleon scattering data or by the validity of approximate flavor $S U(3)$ symmetry. In that case there will be more room for pions, thereby pushing the allowed cutoff to well beyond $1 \mathrm{GeV}$. In other words, we consider the strange sea quarks at moderate $Q^{2}$ as essentially nonperturbative. The early objective of this work was to incorporate kaons and other relevant mesons into the analysis but it was soon recognized that the meson-exchange picture provides a reasonable account for the observed sea quarks in a nucleon at these $Q^{2}\left(\approx 20 \mathrm{GeV}^{2}\right)$ and it is important to confront the model predictions directly with the CCFR results. As we shall see, cutoffs in the range of near $1 \mathrm{GeV}$ when used in our meson-exchange model for parton distributions yields results in good agreement with the CCFR data, thereby not only questioning the validity of the earlier claim [2,3] but offering the validity of using the meson-exchange picture to generate the sea distributions of a hadron at moderate $Q^{2}$.

Perhaps we should clarify our statements a little further. As there is little reason to deny the necessity to include processes as suggested by Sullivan (which scale in the Bjorken limit) nor to deny the successes of using coupling constants given by approximate flavor $S U(3)$ symmetry and relatively hard form factors to obtain excellent fits in a variety of nuclear physics phenomena (such as nucleon-nucleon or hyperon-nucleon scatterings), it is imperative to know how much of the sea content in a hadron can be associated with the meson cloud. It is our finding that, at moderate $Q^{2}$ (say, up to at least $20 \mathrm{GeV}^{2}$ ), the bulk of the sea in a nucleon can be attributed entirely to the meson cloud. Although the gluon splitting process may eventually become important at very large $Q^{2}$ (which we suspect is well beyond $100 \mathrm{GeV}^{2}$ ), we may estimate its importance by noting (on the basis of the QCD evolution equations) that, in going from $Q^{2}=16 \mathrm{GeV}^{2}$ to $100 \mathrm{GeV}^{2}$, the gluon splitting process contributes additional $\delta\langle x\rangle_{s}=(0.2-0.4) \%$ to the existing strange quark sea of slightly more than $1 \%$. The new quarks are preferentially generated at small $x$ which gives rise to an overall "softening" of the parton distributions. On the other hand, in going to smaller $Q^{2}$ the QCD evolution equations give rise to a reduction of sea quarks at small $x$ which makes all the quark distributions 'harder'. The change $\Delta\langle x\rangle^{\text {sea }}$ is less than $10 \%$ if one goes from $Q^{2}=20 \mathrm{GeV}^{2}$ to $Q^{2}=4 \mathrm{GeV}^{2}$ [8]. Such a change of the sea quark distribution appears also in our model, because the QCD evolution equations apply also to the parton distributions of the mesons which are important inputs into our equations. Qualitatively, a 'harder' parton distribution in the mesons give rise to a 'harder' sea quark distribution in baryons. The effects are under investigations.

Thus, there is a very basic difference between what we are trying to do here and that Thomas [2] and Frankfurt et al. [3] have considered. They considered the original Sullivan process as the new source for making additional $\bar{u}$ and $\bar{d}$ sea quarks which are then added to the existing sea distributions of the nucleon. On the other hand, we take out all the sea distributions in the baryons and mesons involved in the "generalized" Sullivan processes and then find that the sea distributions of the nucleon at moderate $Q^{2}$ can even be attributed entirely to the meson cloud.

The significance of our conjecture of attributing the sea distributions of a hadron at moderate $Q^{2}$ to its associated meson cloud as generated by strong interaction processes at the hadron level is that we are now able to determine the parton distributions of a hadron from the knowledge of the valence distributions of the various hadrons, which are calculable from quark models such as a bag model. The QCD evolution equations then take us from moderate $Q^{2}$ to very high $Q^{2}$. The previously very "fuzzy" gap between low $Q^{2}$ (nuclear) physics and large $Q^{2}$ (particle) physics is now linked nicely together, should our conjecture be substantiated by future experiments.

More specifically, we have so far considered the "generalized" Sullivan proceses including those shown in Fig. 2, where the meson and baryon pair $(M, B)$ includes $(\pi, N),(\rho, N),(\omega, N),(\sigma, N),(K, \Lambda),(K, \Sigma),\left(K^{*}, A\right)$,

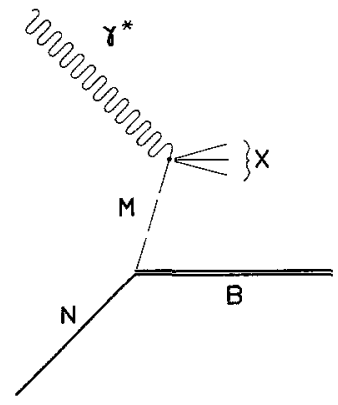

(a)

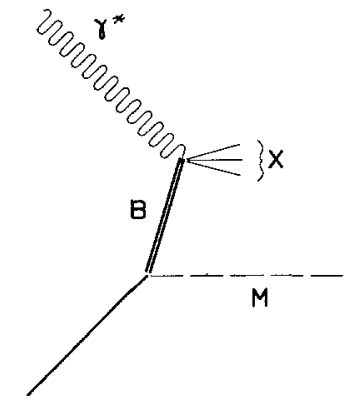

(b)
Fig. $2 \mathbf{a}, \mathbf{b}$. The generalized Sullivan processes a the virtual photon strikes the cloud meson, and $\mathbf{b}$ the virtual photon strikes the recoiling baryons. Both scale in the Bjorken limit. The meson and baryon pair $(M, B)$ includes $(\pi, N),(\rho, N),(\omega, N),(\sigma, N),(K, A)$, $(K, \Sigma),\left(K^{*}, \Lambda\right),\left(K^{*}, \Sigma\right),(\pi, \Delta)$, and $(\rho, \Delta)$ 
$\left(K^{*}, \Sigma\right),(\pi, \Delta)$, and $(\rho, \Delta)$. It turns out that all $f(y)$ 's are such that, as the virtual photon strikes the meson in the "cloud", it sees essentially only the valence partons. Such contributions just look like a "sea" to the nucleon which we thought the experiment would be probing. The value of $R_{\mathscr{Q}}$, which determines the total momentum fraction carried by antiquarks, thus place stringent limits on the form factors associated with the various meson-nucleon strong couplings.

Let us now pause briefly by listing the formulae which we use to obtain our central results. The well-known formula [1] for the Sullivan process is given by

$$
\begin{gathered}
\delta F_{2 N}^{\pi}\left(x, Q^{2}\right)=\int_{x}^{1} \mathrm{~d} y f_{\pi}(y) F_{2 \pi}\left(\frac{x}{y}, Q^{2}\right), \\
f_{\pi}(y)=\frac{3}{4 \pi} \frac{1}{4 \pi}\left(f_{\pi N N} \frac{2 m}{\mu}\right)^{2} y \\
\times \int_{-\infty}^{t^{m}} \mathrm{~d} t \frac{(-t)\left|F_{\pi}(t)\right|^{2}}{\left(-t+\mu^{2}\right)^{2}},
\end{gathered}
$$

where $t^{m}=-m^{2} y^{2} /(1-y)$ with $m$ the nucleon mass. $F_{2 \pi}(x)$ is the pion structure function as would be measured in deep inelastic electron (or muon) scattering. $\delta F_{2 N}(x)$ is the correction to the nucleon structure function due to the Sullivan process. $f_{\pi}(y)$ is the probability of finding a pion carrying the nucleon momentum fraction $y . \mu$ is the pion mass. $f_{\pi N N}$ is the $\pi N N$ coupling in the form of a pseudovector coupling (as dictated by chiral symmetry) with $F(t)$ characterizing its $t$-dependence. As a specific attempt, we consider

$F(t)=\left(\frac{\Lambda_{\alpha}^{2}-m_{\alpha}^{2}}{\Lambda_{\alpha}^{2}-t}\right)^{n_{\alpha}}$.

To simplify the situations, we use $n_{\alpha}=2$ for all couplings and find that a universal cutoff mass of $1150 \mathrm{MeV}$ in the $\Delta / N$ sector and $1400 \mathrm{MeV}$ in the $A / \Sigma$ sector already yields very reasonable results. Nevertheless, we have also considered the cases where all couplings are characterized by exponential cutoffs. In addition, we have also adopted in (3) $n_{\alpha}=1$ for pseudoscalar or scalar mesons and $n_{\alpha}=2$ for vector mesons and for all the couplings in the $\Delta$ sector. Results are similar. In all cases, the quality of the fit to the experiments can be made better by adjusting the various cutoffs.

Introduction of heavy mesons can be made by replacing $f_{\pi}(y)$ by suitable probability functions. For $\rho$, $\omega$, and $\sigma$ mesons, we have obtained, with $\kappa$ the ratio of the tensor to vector coupling,

$$
\begin{aligned}
f_{\rho}(y)= & \frac{3}{2 \pi} \frac{g_{\rho N N}^{2}}{4 \pi}(1+\kappa)^{2} y \\
& \times \int_{-\infty}^{t^{\prime \prime}} \mathrm{d} t \frac{\left|F_{\rho}(t)\right|^{2}}{\left(-t+m_{\rho}^{2}\right)^{2}}\left(-\frac{3}{2} t\right), \\
f_{\omega}(y)= & \frac{1}{2 \pi} \frac{g_{\omega N N}^{2}}{4 \pi}(1+\kappa)^{2} y
\end{aligned}
$$

$$
\begin{aligned}
& \times \int_{-\infty}^{t^{m}} \mathrm{~d} t \frac{\left|F_{\omega}(t)\right|^{2}}{\left(-t+m_{\omega}^{2}\right)^{2}}\left(-\frac{3}{2} t\right), \\
f_{\sigma}(y)= & \frac{1}{4 \pi} \frac{g_{\sigma N N}^{2}}{4 \pi} y \\
& \times \int_{-\infty}^{t^{m}} \mathrm{~d} t \frac{\left|F_{\sigma}(t)\right|^{2}}{\left(-t+m_{\sigma}^{2}\right)^{2}}\left(-t+4 m^{2}\right) .
\end{aligned}
$$

$K N \Lambda, K N \Sigma, K^{*} N \Lambda, K^{*} N \Sigma$ vertices can be introduced analogously except that $t^{m}=m^{2} y-m_{Y}^{2} y /(1-y)$ with $m_{Y}$ the mass of the final hyperon. Note that, in obtaining (4a) and (4b), we sum over only three physical polarizations.

Processes in the type of Fig. 2b, where the virtual photon strikes the recoiling baryon rather than the meson, can be taken into account in a similar fashion. For instance, we obtain, for the $K N A$ vertex,

$$
\begin{aligned}
\delta F_{2 N}^{A}\left(x, Q^{2}\right) & =\int_{x}^{1} \mathrm{~d} y h_{A}(y) F_{2 A}\left(\frac{x}{y}, Q^{2}\right) \\
h_{\Lambda}(y)= & \frac{1}{32 \pi^{2}}\left(f_{K N A} \frac{2 m}{m_{K}}\right)^{2} y \\
& \times \int_{-\infty}^{t^{m}} \mathrm{~d} t \frac{m_{A}}{m^{3}} \frac{\left|\widetilde{F}_{K}(t)\right|^{2}}{\left(-t+m_{A}^{2}\right)^{2}} \\
& \times\left\{-m_{A} m m_{K}^{2}+\frac{1}{2}\left(\left(m^{2}-t\right)^{2}-m_{K}^{2}\left(m^{2}+t\right)\right)\right\} .
\end{aligned}
$$

Here $t^{m}=m^{2} y-m_{K}^{2} y /(1-y)$ and the form factor $\tilde{F}(t)$ is now somewhat unusual:

$$
\tilde{F}(t) \equiv \frac{f_{K N A}\left(-m_{K}^{2} ;-t,-m^{2}\right)}{f_{K N A}\left(-m_{K}^{2} ;-m_{\Lambda}^{2},-m^{2}\right)} .
$$

And, for the $K^{*} N \Lambda$ and $\sigma N N$ vertices,

$$
\begin{aligned}
h_{\Lambda}^{*}(y)= & \frac{1}{8 \pi^{2}} g_{K^{*} N \Lambda}^{2} y \\
& \times \int_{-\infty}^{t^{m}} \mathrm{~d} t \frac{m_{A}}{m} \frac{\left|\tilde{F}_{K^{*}}(t)\right|^{2}}{\left(-t+m_{A}^{2}\right)^{2}} \\
& \times\left\{m_{\Lambda}\left(-3 m-\frac{\kappa}{2 m} 3 t-\frac{\kappa^{2}}{4 m} 3 t\right)\right. \\
& +\left(-3\left(t-m^{2}\right)-\kappa \frac{3}{2} t-\kappa^{2} \frac{3}{4} t\right\} . \\
h_{N}^{\sigma}(y)= & \frac{1}{8 \pi^{2}} g_{\sigma N N}^{2} y \\
& \times \int_{-\infty}^{t^{m}} \mathrm{~d} t \frac{\left|\tilde{F}_{\sigma}(t)\right|^{2}}{\left(-t+m^{* 2}\right)^{2}} \frac{1}{2}\left\{3 m^{2}-m_{\sigma}^{2}+t\right\} .
\end{aligned}
$$

Note that, in obtaining the $h(y)$ functions, we use $\mathrm{i} \gamma \cdot p u(p)=m u(p)$ (Dirac equation) for the recoiling baryon. Ambiguities arising from the off-shellness of the recoiling baryon remain to be clarified although, as far 
as we can tell, it does not affect the results reported here in any significant manner. In addition, we wish to remark that, since the final meson, instead of the recoiling baryon, must be on-shell for processes illustrated by Fig. $2 \mathrm{~b}$, the functions $h_{i}(y)$ are related, but not identical, to the corresponding $f_{i}(y)$. Except perhaps for strange particles, it is unlikely that a highly virtual recoiling baryon such as $\Delta^{*}$, as accompanied by emission of a meson, would survive long enough to be struck by the probing (virtual) photon.

As for the $\pi N \Delta$ and $\rho N \Delta$ vertices, we adopt the chiral Lagrangians $[9,10]$ in terms of Rarita-Schwinger spinors. (See [10] for the notations which we adopt here.) The $f(y)$ and $h(y)$ functions for the $\pi N \Delta$ vertex are also recorded here for reference,

$$
\begin{aligned}
f_{\pi}^{\Delta}(y)= & \frac{1}{12 \pi^{2}} g_{\Delta}^{2} y \\
& \times \int_{-\infty}^{t^{m}} \mathrm{~d} t \frac{1}{\mu^{2}} \frac{\left|F_{\pi N \Delta}(t)\right|^{2}}{\left(-t+\mu^{2}\right)^{2}} \\
& \times \frac{1}{2}\left\{\left(m+m_{\Delta}\right)^{2}-t\right\} \\
& \times\left\{-m^{2}+\frac{1}{4 m_{\Delta}^{2}}\left(m^{2}+m_{\Delta}^{2}-t\right)^{2}\right\},
\end{aligned}
$$

$$
\begin{aligned}
h_{\Delta}^{\pi}(y)= & \frac{1}{12 \pi^{2}} g_{\Delta}^{2} y \\
& \times \int_{-\infty}^{t^{m}} \mathrm{~d} t \frac{m_{\Delta}}{\mu^{2} m} \frac{\left|\tilde{F}_{\pi N \Delta}(t)\right|^{2}}{\left(-t+m_{\Delta}^{2}\right)^{2}} \\
& \times\left\{\frac{1}{2}\left(t+m^{2}-\mu^{2}\right)+m m_{\Delta}\right\} \\
& \times\left\{-\mu^{2}+\frac{1}{4 m_{\Delta}^{2}}\left(t-m^{2}+\mu^{2}\right)^{2}\right\} .
\end{aligned}
$$

At the first sight, there might appear a good number of parameters in our model. This is in fact not true. All the coupling constants and masses are taken to be the same as those obtained or used in [7]. Although it is obvious $[2,3]$ that the predictions are extremely sensitive to the various cutoffs (or factors), we decide after a few numerical trials to set all the $t$-channel cutoff masses $\Lambda_{\alpha}$ 's in the $\Delta / N$ and $A / \Sigma$ sectors respectively to universal values of $1150 \mathrm{MeV}$ and $1400 \mathrm{MeV}$. In the case of mixed monopole-dipole scenario, we also set all the $t$-channel cutoffs to a universal value $M_{A}$ except the pion cutoff $\Lambda_{\pi}$ which is treated as a parameter. Here the value $M_{A}$ is taken to be the one which governs the $q^{2}$ dependence of the axial form factors (in dipole form) and is known to be $1032( \pm 0.036) \mathrm{MeV}$ experimentally [12]. Reasonable results are obtained with $A_{\pi}=950 \mathrm{MeV}$ or at a slightly smaller value. The timelike form factors as defined by (6) are taken to be of the same form as the corresponding $t$-channel ones (with $m_{\alpha}$ now identified with the recoiling baryon mass) except that the cutoff masses are adjusted harder by a universal rescaling factor $\gamma$. To ensure that, in a free nucleon, the model generates an equal amount of strangeness and antistrangeness, we fix the value of $\gamma$. This factor is also used in the $\Delta$ and $N$ sectors. When all couplings are characterized by exponential cutoffs, we adopt universal values of $900 \mathrm{MeV}$ and $1150 \mathrm{MeV}$ respectively for the $N / \Delta$ and $\Lambda / \Sigma$ sectors.

On the other hand, the structure functions for the pions, especially the valence distributions which are the essential input information, are known experimentally [12]. Using flavor $S U(3)$ rotations while taking spinisospin average, we obtain structure functions for all the other mesons. We take into consideration the slight difference [13] between the kaon and pion valence distributions. For the structure functions of a nucleon, we have considered distributions obtained by Duke and Owens [14] and by Eichten et al. [15] (EHLQ). At $Q^{2}=16.85 \mathrm{GeV}^{2}$, we find that, while the sea content of the Duke-Owens distributions is too strong in comparison with the CCFR data (1), the EHLQ distributions are in good agreement with the CCFR data. As we shall need only the valence and glue distributions in our theory, however, both distributions give rise to very similar results. The distribution functions for $\Lambda, \Sigma$ and $\Delta$ are then obtained again by flavor $S U(3)$ rotations. Small breaking of flavor $S U(3)$ symmetry does not affect our major conclusions.

To test our conjecture that the sea quarks of the nucleon can be generated entirely by the meson-exchange picture, we take out the sea distributions in all baryons and carry out the convolution indicated by (2a) and (5a). In addition to the sea quarks which can be generated in this manner, we also expect to have renormalizations over the valence distributions. For example, kaons provide $u$ or $d$ quarks in addition to $\bar{s}$ quarks. Thus we renormalize the original valence distributions before we add the "sea" to it, in order to make certain that the number sum rules are maintained. In the same spirit, we renormalize the original glue distributions before we add the new "sea glue" to it, so that the momentum sum rules are satisfied by ansatz.

The predictions of the meson-exchange model (in the dipole form) are listed below (with the results using exponential cutoffs for all couplings or the mixed monopole-dipole scenario listed in parentheses). The EHLQ valence and glue distributions are used but the results from using Dukes-Owens distributions differ by at most a few percent.

$$
\kappa \equiv \frac{2\langle x\rangle_{s}}{\langle x\rangle_{\bar{u}}+\langle x\rangle_{\bar{d}}}=0.40
$$

$$
\text { (Expo: 0.40; Mixed: 0.39), }
$$

$$
\eta_{s} \equiv \frac{2\langle x\rangle_{s}}{\langle x\rangle_{u}+\langle x\rangle_{d}}=0.056
$$

(Expo: 0.054; Mixed: 0.062),

$$
R_{\bar{Q}} \equiv \frac{\langle x\rangle_{\bar{u}}+\langle x\rangle_{d}+\langle x\rangle_{\bar{s}}}{\langle x\rangle_{u}+\langle x\rangle_{d}+\langle x\rangle_{s}}=0.161
$$

(Expo: 0.160; Mixed: 0.189) 

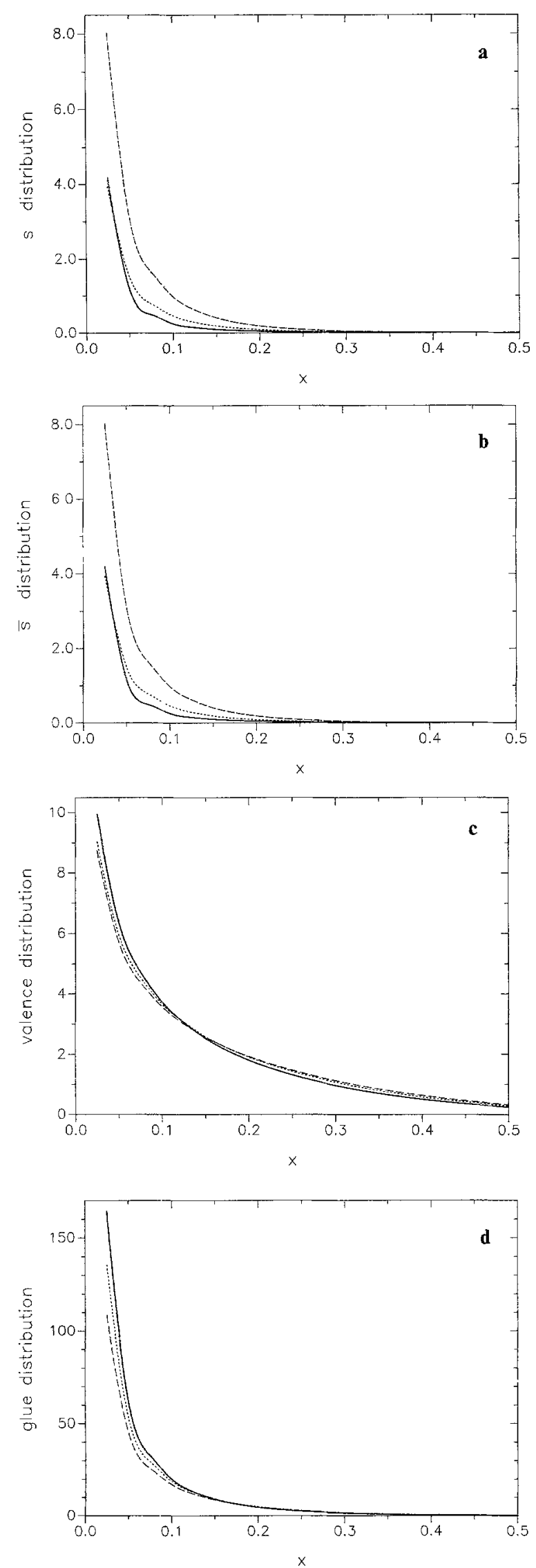

Fig. 3a-d. The calculated parton distributions (in solid curves), as explained in the text, and the corresponding ones from EHLQ (in dotted curves) and Duke-Owens (in dashed curves) at $Q^{2}=16.85 \mathrm{GeV}$ plotted as functions of $x$

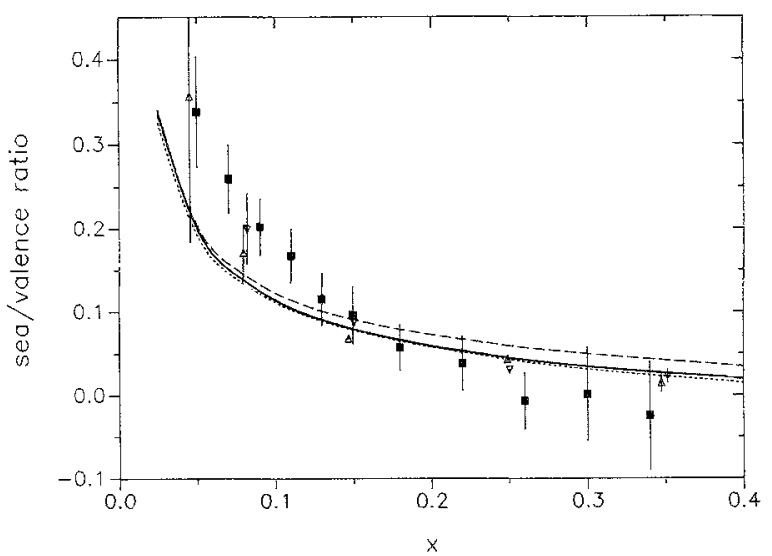

Fig. 4. The ratio $\frac{1}{2}\{\bar{u}(x)+\bar{d}(x)\} /\left\{u_{v}(x)+d_{v}(x)\right\}$ shown as a function of $x$ for $Q^{2}=16.85 \mathrm{GeV}^{2}$, which may be compared with, e.g., the sea-to-valence ratio extracted from the CDHS data (in triangles) [16] and the E615 data (in solid squares) [17]. The solid curve is obtained by using dipole form factors for all couplings; the dotted curve is for exponential cutoffs; and the dashed curve is for the mixed monopole-dipole scenario

which, even without any serious attempt to obtain the best fit, are in excellent agreement with the CCFR data, (1a)-(1c).

It is also remarkable to find that the shape of the various parton distributions obtained in this way are very similar to that in the EHLQ distributions at $Q^{2}=16.85 \mathrm{GeV}^{2}$, lending support toward our conjecture that sea distributions of a hadron at moderate $Q^{2}$ come almost entirely from the meson cloud. The distributions obtained in our model (denoted by solid curves) and the corresponding ones from EHLQ (in dotted curves) and Duke-Owens (in dashed curves) (at $Q^{2}=16.85 \mathrm{GeV}$ ) are shown as functions of $x$ in Figs. $3 \mathrm{a}-3 \mathrm{~d}$, where the $s$-quark, $\bar{s}$-quark, valence quark, and glue distributions are plotted respectively as functions of $x$.

Nevertheless, our conjecture can be subject to experimental tests in the immediate future. For instance, we plot in Fig. 4 the ratio $\frac{1}{2}\{\bar{u}(x)+\bar{d}(x)\} /\left\{u_{v}(x)+d_{v}(x)\right\}$ as a function of $x$ for $Q^{2}=16.85 \mathrm{GeV}^{2}$ which may be compared with, e.g., the sea-to-valence ratio extracted from the CDHS data (in triangles) [16] and the E615 experiment (in solid squares) [17]. Here the solid curve is obtained by using dipole form factors for all couplings; the dotted curve is for exponential cutoffs; and the dashed curve is for the mixed monopole-dipole scenario. It is clear that such comparison suggests adoption of scenarios in which the form factors falls off faster than the mixed monopole-dipole scenario. We have considered the relative importance of the contributions from different sectors by varying individual cutoff $A_{\alpha}$. The results indicate, for example, that the contribution from the $\pi N \Delta$ vertex falls off slightly faster than that from the $\pi N N$ vertex, but such difference is considerably smaller than those among the three curves shown in the figure.

There is an excellent agreement between our prediction (e.g. the solid curve) and the CDHS data [16], both with $Q^{2}$ in the vicinity of $16 \mathrm{GeV}^{2}$. Our results are also in good agreement with the EHLQ prediction. Neverthe- 
less, all of these predictions (including EHLQ) at very small $x$ are somewhat too low as compared with the E615 data [17] in which the quantity $\frac{1}{2}\left\{u_{s}(x)\right.$ $\left.+d_{s}(x)\right\} /\left\{u_{v}(x)+d_{v}(x)\right\}$ was measured in the $Q^{2}$ range $20 \mathrm{GeV}^{2}<Q^{2}<70 \mathrm{GeV}^{2}$. The $\mathrm{E} 615$ data agrees with the expectation from the Duke-Owens distribution, which is however at odd with the recent CCFR data. It is not clear if the discrepancy between our prediction and the E615 data at small $x$ can be attributed to the difference in $Q^{2}$ since the $Q^{2}$ dependence of the pion valence distributions is not known and has so far been left out in our calculations. It is also not clear if utilization of a nuclear target (tungsten in E615) in extracting the sea-to-valence ratio poses some problem in light of the so-called EMC effect [18] (i.e. modification of the nucleon structure in nuclei). (However, the CDHS data are also from neutrino scattering with a nuclear target, iron.) In any event, consistency among the data for extracting pion distributions, the CCFR data, and the earlier CDHS data emerges nicely within our model calculations.

Nevertheless, presence of the Sullivan processes invalidates the naive counting rules as $x \rightarrow 1$ or $x \rightarrow 0$ such that sea distributions in our model behave somewhat differently near $x=1$ or $x=0$ from the phenomenological distributions such as EHLQ or Duke-Owens. Such differences, which are not visible except at large $x$ (near $x=1$ ) or very small $x$ (say, less than 0.05 ), may offer clues in testing our conjecture.

As a footnote, we find that increase of $\Lambda_{\pi}$ (in the monopole form) from $950 \mathrm{MeV}$ to $1200 \mathrm{MeV}$ (or even higher) would still fit very well the value of $\kappa$ as long as $A_{K}$ is adjusted to a higher value accordingly, indicating the shortcomings of the argument of the previous work $[2,3]$. However, the sea distributions would be completely at odds with the value of $R_{\bar{Q}}$, since we would have too much antiquark sea. We may also note that a pion monopole form factor of $950 \mathrm{MeV}$ is already much harder than the value advocated in [2] and [3]. In terms of the momentum fraction carried by pions, our result at $950 \mathrm{MeV}$ is $\langle y\rangle_{\pi}=19 \%$, significantly higher than the upper limits of $(12.4 \pm 3.3) \%$ and $(5.0 \pm 1.5) \%$ adopted by Frankfurt et al. [3] and Thomas [2], respectively, in reaching their conclusions. All these numbers, however, are appreciably larger than the value of $\langle y\rangle_{\pi^{+}}=(0.6 \pm 0.2) \%$ by Güttner et al. [19] which has been deduced from semi-inclusive meson production. Using the translation formula used by Thomas [2], the bag radius corresponding to $\langle y\rangle_{\pi}=19 \%$ is about $0.49 \mathrm{fm}$.

Interference processes where a photon interacts with antiquarks from different mesons or those between a photon interacting with quarks in the meson cloud and the nucleon have not been included in this work nor in that of [2] and [3]. As the number of pions per nucleon, here $N_{n / N} \approx 0.6$, is larger than in the previous approaches, the interference effects might also be somewhat larger. These contributions should be considered in future refinements of meson-nucleon form-factor determinations. In [3] arguments are given that such interference effects appear only at very small $x$-values and so they would have little influence on our conclusion.
In obtaining our results, we had to adjust the cutoffs to values below those used for fitting the nucleon-nucleon and hyperon-nucleon scattering data. This of course destroys the existing fits. With now the various cutoffs constrained by the deep inelastic scattering data and with the coupling constants (previously fixed to the $S U(3)$ values) adjusted slightly to allow for small flavor $S U(3)$ symmetry breaking, fits of similar quality can still be obtained. However, there is a difference, compared to the original approaches where only the lowest mass mesons have been considered. If one uses e.g. for monopole formfactors a cut-off of $A=800 \mathrm{MeV}$ for a given meson one has to introduce an additional heavy mass meson with the same quantum numbers $[20,21]$. The parameters of such mesons are purely phenomenological. These additional contributions may be considered as an average effect of all the higher mass mesons. But, from our point of view, it is more likely, that they simulate the interaction between the valence quarks of the two nucleons. Such "quark effects" give rise to an interaction which looks very similar to the one obtained from a one boson exchange model [22]. Therefore we may conclude: if one uses for meson exchange potentials formfactors which are consistent with deep inelastic scattering, one has to introduce additional contributions which may reflect the so called "quark-effects". However, we should bear in mind that the deep inelastic scattering data places stringent constraints only for certain cutoffs in the $\pi, \rho$, and $K$ sectors but still say very little for certain vertices such as $\omega N N, \sigma N N$, etc.

We may use our model to determine possible deviation from the Gottfried sum rule. In the dipole scenario, we obtain

$$
\int_{0.05}^{1} \frac{\mathrm{d} x}{x}\left\{F_{2}^{e p}(x)-F_{2}^{e n}(x)\right\}=0.25,
$$

which compares well with a preliminary value [27] reported by the NMC group:

$$
\begin{aligned}
& \int_{0.04}^{1} \frac{\mathrm{d} x}{x}\left\{F_{2}^{e p}(x)-F_{2}^{e n}(x)\right\} \\
& \quad=0.218 \pm 0.008 \text { (stat.) } \pm 0.021 \text { (syst.). }
\end{aligned}
$$

In fact, a better agreement can easily be obtained by either increasing the cutoff in the nucleon sector by about $50 \mathrm{MeV}$, or decreasing that in the $\Delta$ sector by a slightly larger amount, while maintaining the quality of the agreement between the model predictions and the data mentioned in the text.

An important aspect of nuclear physics is that quark distributions of nucleons in nuclei are expected to be different from quark distributions of nucleons in free space. Several phenomena are present in nuclei which can lead to modified quark distributions as measured by deep inelastic lepton-nucleus scattering [18] or lepton pair production [23] in hadron-nucleus collisions.

Our meson-exchange model for nucleon parton distributions provides a framework for understanding how 
parton distributions of a nucleon are modified in a nucleus. Our model might be considered as a generalization of the pion-excess model [24] but one may argue [25] how different hadron properties are rescaled in a universal manner, resulting in cancellation of the "unwanted" effects in nuclear medium. For instance, there is indication that inclusion of effects illustrated by Fig. $2 b$ yields a "shadowing effect" at very small $x$. Results of such further investigations are to be reported elsewhere [26].

In summary, we have introduced a meson-exchange model for understanding the sea distributions of a nucleon at moderate $Q^{2}$, by taking into account effects of the various mesons including $\pi, \rho, \omega, \sigma, K$, and $K^{*}$, with the coupling constants fixed by the low-energy nucleonnucleon and hyperon-nucleon scattering data. Contrary to an earlier claim that the $\pi N N$ and $\pi N \Delta$ form factor must be very soft (e.g., with the cutoff mass less than $500 \mathrm{MeV}$ in the monopole form), we have found, for example, that, with all form factors parametrized in the dipole form, a universal cutoff mass of $1150 \mathrm{MeV}$ in the $\Delta / N$ sector and $1400 \mathrm{MeV}$ in the $\Lambda / \Sigma$ sector yields predictions in excellent agreement with the CCFR results (1a)-(1c). When exponential cutoffs are adopted for all couplings, the results are very similar. In the mixed monopole-dipole scenario, a pion cutoff mass of about $950 \mathrm{MeV}$ in the monopole form and a universal cutoff of slightly above $1000 \mathrm{MeV}$ for all the other meson-baryon strong couplings yields reasonable results. In all cases, the parton distributions obtained in our model are very similar to the corresponding ones in existing parametrized parton distributions such as Eichten et al. [15].

We wish to thank G. Garvey, K. Holinde, L.-C. Liu, M. B. Johnson, M. Strikman, A. W. Thomas and Jochen Wambach for helpful conversations. W-Y. P. Hwang wishes to acknowledge the Alexander von Humboldt Foundation for a fellowship to visit Jülich for conducting research. His research works was also supported in part by the National Science Council of the Republic of China. The work of G. E. Brown is supported in part by a Humboldt award, in part by NATO Grant RG85/0093, and in part by the U.S. Department of Energy.

\section{References}

I. Sullivan, J.D.: Phys. Rev. D5, 1732 (1972)

2. Thomas, A.W.: Phys. Lett. 126B, 97 (1983)
3. Frankfurt, L.L., Mankiewicz, L., Strikman, M.I.: Z. Phys. A Atomic Nuclei 334, 343 (1989)

4. Foudas, C. et al.: CCFR Coll., Phys. Rev. Lett. 64, 1207 (1990)

5. MacFarlane, D. et al.: Z. Phys. C - Particles and Fields 26, 1 (1984); Oltman, E.: In: The Storrs Meeting: Proceedings of the Division of Particles and Fields of the American Physical Society, 1988, Hall, K. et al. (eds.). Singapore: World Scientific 1989

6. Field, R.D., Feynman, R.P.: Phys. Rev. D15, 2590 (1977)

7. Holzenkamp, B., Holinde, K., Speth, J.: Nucl. Phys. A500, 485 (1989)

8. Harriman, P.N., Martin, A.D., Sterling, W.J., Roberts, R.G.: Phys. Rev. D42, 758 (1990)

9. Peccei, R.D.: Phys. Rev. 176, 1812 (1968); 181, 1902 (1969)

10. Hwang, W-Y.P., Walker, G.E.: Ann. Phys. (New York) 159, 118 (1985)

11. Ahrens, L.A. et al.: Phys. Rev. D35, 785 (1987)

12. Badier, J. et al.: Z. Phys. 18, 281 (1983)

13. Badier, J. et al. : Phys. Lett. 93B, 354 (1980)

14. Duke, D.W., Owens, J.F.: Phys. Rev. D30, 49 (1984)

15. Eichten, E., Hinchliffe, I., Lane, K., Quigg, C.: Rev. Mod. Phys. 56, 579 (1984); Erratum: 58, 1065 (1986)

16. Abramowicz, H. et al.: CDHS Coll., Z. Phys. C - Particles and Fields 17. 283 (1983)

17. Heinrich, J.G. et al.: E615 Coll., Phys. Rev. Lett. 63, 356 (1989)

18. Aubert, J.J. et al.: Phys. Lett. 123B, 275 (1983); Landgraf, U., New Muon Coll. : Nuclear Effects in Deep Inelastic Scattering. Talk at PANIC XII, M.I.T. 1990

19. Güttner, E., Chanfray, G., Pirner, H.J., Povh, B.: Nucl. Phys. A 429, 389 (1984)

20. Holinde, K., Thomas, A.W.: Phys. Rev. C42, 1195 (1990) and private communications

21. Deister, S., Gari, M., Krümpelmann, W., Mahlke, M.: Preprint and private communications

22. Holinde, K.: Phys. Lett. 111 B, 266 (1982)

23. Alde, D.M. et al.: E772 Coll. : Phys. Rev. Lett. 64, 2749 (1990)

24. Llewellyn Smith, C.H.: Phys. Lett. 128B, 107 (1983); Ericson, M., Thomas, A.W.:Phys. Lett. 128B, 112 (1983); Ericson, M.: Erice lectures 1983, Prog. Part. Nucl. Phys. 11, 277 (1984); Thomas, A.W.: Erice lectures 1983, Prog. Part. Nucl. Phys. 11, 325 (1984); Berger, E.L., Coester, F., Wiringa, R.B.: Phys. Rev. D29, 398 (1984); Stump, D., Bertsch, G.F., Pumplin, J.: Hadron Substructure in Nuclear Physics, p. 339. New York: American Institute of Physics 1984

25. Brown, G.E.: Matter under extreme conditions. Talk at the Arima Conference, Santa Fe, New Mexico 1990

26. Hwang, W-Y.P., Speth, J., Wambach, J., Brown, G.E.: Work in progress

27. Windmolders, R., NMC Coll.: Contribution to PANIC XII, M.I.T., 1990 